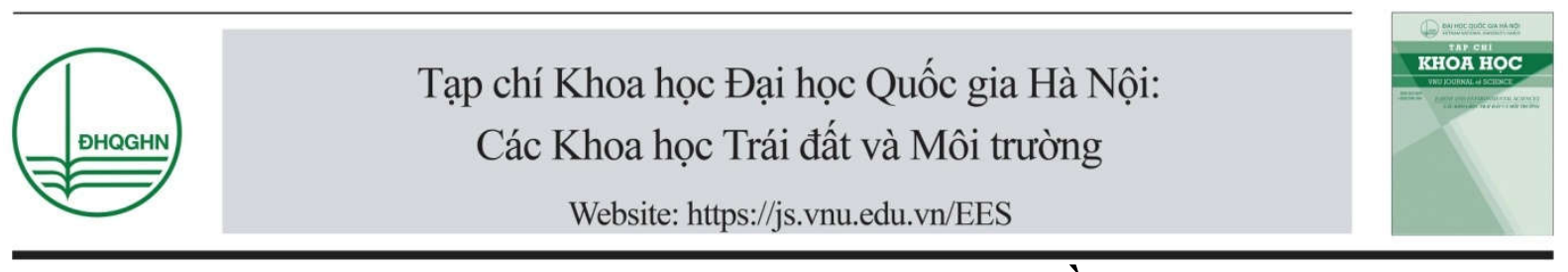

Đánh giá ước lượng mưa từ độ phản hồi radar Nhà Bè

\author{
Công Thanh ${ }^{1, *}$, Nguyễn Như Quý ${ }^{1}$, Mai Văn Khiêm² \\ ${ }^{I}$ Khoa Khi tuợng Thủy văn và Hải dương học, Trương Đại học Khoa học Tụ nhiên, ĐHQGHN, \\ 334 Nguyễn Trãi, Thanh Xuân, Hà Nội, Việt Nam \\ ${ }^{2}$ Viện Khoa học Khi tuợng Thủy văn và Biến đổi khí hậu, \\ 23/62 Nguyễn Chí Thanh, Đống Đa, Hà Nọii, Việt Nam \\ Nhận ngày 30 tháng 11 năm 2018 \\ Chỉnh sửa ngày 10 tháng 12 năm 2018; Chấp nhận đăng ngày 25 tháng 12 năm 2018
}

\begin{abstract}
Tóm tắt: Nghiên cứu này đánh giá ước lượng mưa từ quan trắc radar Nhà Bè theo các công thức thực nghiệm khác nhau. Kết quả cho thấy, trong số 6 công thức ước lượng được sử dụng, công thức của ước lượng mưa diện rộng của Joss và công thức của Marshall-Plamer có thể được sử dụng để ước lượng mưa cho hình thế gió mùa tây nam đơn thuần với sai số vào khoảng là $5 \mathrm{~mm} / \mathrm{h}$ và thường nhỏ hơn giá trị quan trắc. Công thức ước lượng mưa do Nguyễn Hướng Điền (2015) [1] đưa ra là ước lượng tối ưu cho 9 hình thế gây mưa: dải hội tụ nhiệt đới, gió mùa tây nam kết hợp dải hội tụ nhiệt đới, vùng áp thấp đơn thuần, dông nhiệt, nhiễu động gió đông, vùng áp thấp kết hợp với gió mùa tây nam, rãnh áp thấp có hướng tây bắc-đông nam và các hình thế khác với sai số lần lượt là $9.5 \mathrm{~mm} / \mathrm{h}, 14 \mathrm{~mm} / \mathrm{h}, 7 \mathrm{~mm} / \mathrm{h}, 19 \mathrm{~mm} / \mathrm{h}, 8 \mathrm{~mm} / \mathrm{h}, 8.5 \mathrm{~mm} / \mathrm{h}, 9.5 \mathrm{~mm} / \mathrm{h}$ và $14.7 \mathrm{~mm} / \mathrm{h}$.
\end{abstract}

Tù khóa: Radar, ước lượng mưa.

\section{Mở đầu}

Trong những năm gần đây, radar đã trở thành công cụ rất quan trọng trong quan trắc và dự báo thời tiết như dông, bão, mưa lớn,... Tại cuộc hội thảo tập huấn về tiêu chuẩn chất lượng dữ liệu radar thời tiết được tổ chức tại Thái Lan [2] đã nêu ra những vấn đề về việc theo dõi và dự báo thời tiết nguy hiểm đặc biệt là kiểm soát chất lượng và ước lượng lượng mưa $(\mathrm{QPF})$ từ độ phản hồi radar cho khu vực Đông Nam Á. Trong buổi hội thảo, họ nhấn mạnh tầm quan trọng của mạng lưới radar và xem xét tăng

\footnotetext{
* Tác giả liên hệ. ĐT.: 84-946180348.

Email: thanhc@vnu.edu.vn

https://doi.org/10.25073/2588-1094/vnuees.4330
}

cường hệ thống radar thời tiết để nâng cao chất lượng dự báo thời tiết. Để ước lượng mưa từ số liệu quan trắc radar, rất nhiều công thức thực nghiệm đã được phát triển dựa trên tương quan hệ giữa mưa và độ phản hồi radar Z. Mỗi công thức thực nghiệm có ưu và nhược điểm khác nhau. Để có thể ứng dụng vào dự báo nghiệp vụ thì các công thức thực nghiệm cần được kiểm định và hiệu chỉnh các hệ số thực nghiệm. Radar có thể cung cấp các thông tin định lượng về lượng mưa với độ phản hồi radar từ mưa, biến đổi từ $20 \mathrm{dBz}$ đến hơn $50 \mathrm{dBz}$. Độ phản hồi cao có thể đạt đến $75 \mathrm{dBz}$ trong mưa dông, nhưng độ phản hồi cao trên $55 \mathrm{dBz}$ lại thường gắn liền với mưa đá. Mỗi loại mưa có một hàm phân bố hạt theo kích thước riêng, người ta đã 
xác định nhiều cặp giá trị $\mathrm{a}$ và $\mathrm{b}$ cho từng loại mưa. Battan (1973) [3] đã liệt kê trên 60 quan hệ $\mathrm{Z}-\mathrm{R}$. Mỗi phương trình thích hợp với từng hoàn cảnh cá biệt. Hầu hết các quan hệ này không khác nhau nhiều khi cường độ mưa nằm trong khoảng từ 20 đến xấp xỉ $200 \mathrm{~mm} / \mathrm{h}$. Nghiên cứu gần đây của Nguyễn Hướng Điền (2015) [1]. Dựa trên các số liệu độ phản hồi của radar thời tiết đặt tại Nhà Bè và lượng mưa quan trắc được tại 4 trạm đo mưa mặt đất (Tân Sơn Hòa, Chơn Thành, Lộc Ninh và Tây Ninh) trong các đợt mưa diện rộng trong các năm 2010-2012, tác giả đã đưa ra công thức thực nghiệm ước lượng mưa cho khu vực Đông Nam Bộ. Kết quả nghiên cứu này cho thấy Các công thức lấy $\mathrm{R}$ là hàm có các giá trịsai số $\mathrm{ME}$ nhỏ hơn nhiều so với các giá trịtương ứng của công thức lấy $\mathrm{Z}$ là hàm và Marshall-Palmer khi đã thây đổi các hệ số $A, B$ và sai số $M E, M E$, RMSE đối với công thức này lần lượt là là 0.59 ; 3.56; 7.68 cho trạm Tân Sơn Hòa. Tác giả cũng kết luận công thứ dạng $\mathrm{R}$ là hàm có độ chính xác cao hơn hẳn các dạng công thức khác.

Tuy nhiên, có những trường hợp rất khó xếp vào loại nào như mưa hỗn hợp (lỏng lẫn với đá, tuyết). Một số công thức thực nghiệm điển hình thể hiện mối quan hệ giữa độ phản hồi radar và cường độ mưa với các kiểu mưa khác nhau được cho trong bảng 1 .

Bảng 1. Một số công thức thực nghiệm điển hình thể hiện mối quan hệ giữa độ phản hồi $\mathrm{Z}\left(\mathrm{mm}^{6} / \mathrm{m}^{3}\right)$ và cường độ mưa $\mathrm{R}(\mathrm{mm} / \mathrm{h})[3]$

\begin{tabular}{|c|c|c|}
\hline Quan hệ thực nghiệm Z-R & Kiểu mưa & Tác giả \\
\hline$Z=140 R^{1.5}$ & Mưa phùn & Joss và cộng sự (1970) \\
\hline$Z=250 R^{1.5}$ & Mưa diện rộng & Joss và cộng sự (1970) \\
\hline$Z=200 R^{1.6}$ & Mưa dầm & Marshall và Palmer (1948) \\
\hline$Z=31 R^{1.71}$ & Mưa địa hình & Blanchard (1953) \\
\hline$Z=500 R^{1.5}$ & Mưa dông & Joss và cộng sự (1970) \\
\hline$Z=486 R^{1.37}$ & Mưa dông & Jones (1956) \\
\hline$Z=2000 R^{2.0}$ & Mưa bông tuyết lớn & Gunn và Marshall (1958) \\
\hline$Z=1780 R^{2.21}$ & Mưa tuyết & Sekhon và Srivastava (1970) \\
\hline
\end{tabular}

Mỗi công thức thực nghiệm có ưu và nhược điểm khác nhau. Để có thể ứng dụng vào dự báo nghiệp vụ thì các công thức thực nghiệm cần được kiểm định và hiệu chỉnh các hệ số thực nghiệm. Nghiên cứu này đánh giá ước lượng mưa từ quan trắc radar Nhà Bè theo các công thức thực nghiệm khác nhau cho một số hình thế thời tiết điển hình thường gây mưa lớn ở khu vực Nam Bộ nhằm mục đích tìm công thức tối ưu cho các hình thế thời tiết phục vụ bài toán ước lượng lượng mưa từ sản phẩm ngoại suy độ phản hồi radar sau này. Trong mục 2 của bài báo trình bày số liệu và mô tả phương pháp nghiên cứu, mục 3 là kết quả và thảo luận.

\section{Số liệu và phương pháp nghiên cứu}

\subsection{Số liệu quan trắc mưa}

Số liệu quan trắc mưa bề mặt được thu thập từ 26 trạm đo mưa tự động, trạm khí tượng và trạm đo mưa truyền thống trong khu vực thành phố Hồ Chí Minh và các tỉnh lân cận. Các trạm này bao gồm các trạm: Cần Giờ, Củ Chi, Quận 12, Tân Thông Hội, Bình Tân, Quận 7, Quận 8, Tân Sơn Hòa, Nhà Bè, Mạc Đĩnh Chi, Tân An, Mỹ Tho, Sở Sao, Biên Hòa, Vũng Tàu, Tân Sơn Nhất, Thanh Đa, Lý Thường Kiệt, Quang Trung, Cầu Bông, Phước Long, Phan Văn Khỏe, Tây Ninh, Mộc Hóa. Số liệu sau khi thu thập được phân tích và đánh giá theo các hình thế gây mưa chủ yếu trong mùa mưa của khu vực Nam Bộ trong các năm 2013, 2014 và 2015.

\subsection{Số liệu radar thời tiết}

Số liệu ra đa thời tiết dùng để ước lượng mưa chúng tôi sử dụng $\mathrm{CAPPI}(\mathrm{Z}) 1 \mathrm{~km}$, với bán kính quét $240 \mathrm{~km}, 480 \mathrm{~km}$ và chu kỳ quan trắc, lưu số liệu từ $5-30$ phút/lần. Trong đó ý nghĩa của các sản phẩm được lưu trữ dưới dạng các file dữ liệu được sắp xếp theo trật tự thời gian của từng trạm. 


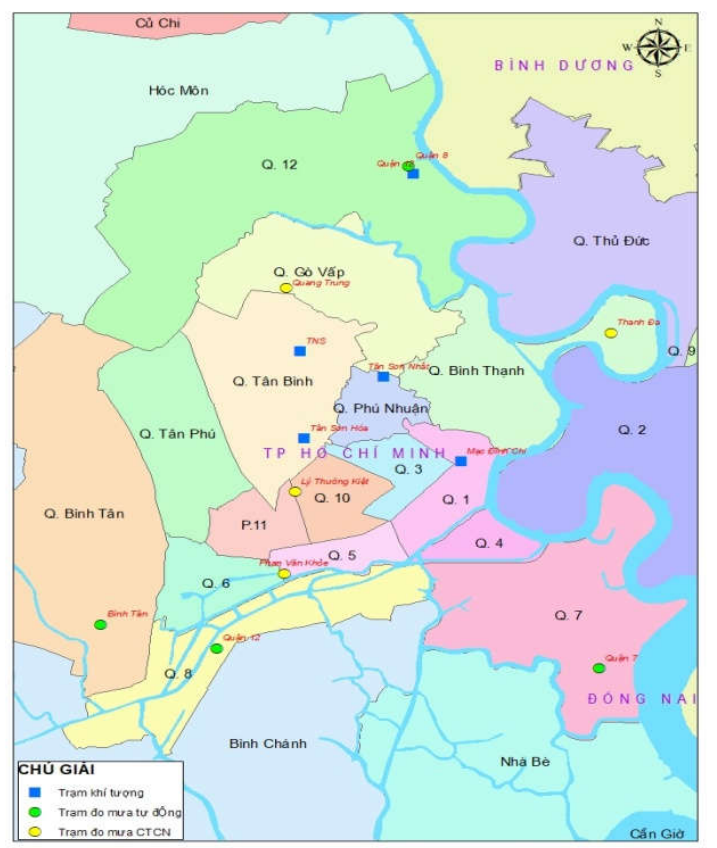

Hình 1. Các trạm đo mưa ở Thành phố Hồ Chí Minh.

Cụ thể: Số liệu ước lượng mưa được tính từ CAPPI $1 \mathrm{~km}$ với các công thức: công thức Marshall, công thức được đưa ra bởi Nguyễn Hướng Điền, công thức cho mưa dông của Joss, công thức cho mưa dông của Jones, công thức cho mưa diện rộng của Joss và ước lượng mưa trực tiếp từ VIL.

\subsection{Các công thức tính ước lương mưa sủ dụng:}

Marshall-Palmer:

$$
Z=200 R^{1.6}
$$

Công thức đưa ra bởi Nguyễn Hướng Điền (VNU): [2] $R=4.4742805 * 10^{0.022283 * Z^{\prime}}$

Công thức mưa dông của Joss: [5]

$$
Z=500 R^{1.5}
$$

Công thức mưa dông của Jones:

$$
Z=486 R^{1.37}
$$

Công thức mưa diện rộng của Joss: [5]

$$
Z=200 R^{1.5}
$$

Uớc lượng trực tiếp từ VIL theo nguyên tắc lượng giáng thủy bằng độ hụt khối lượng nước tích trữ trong cột khí quyển.

\subsection{Phuơng pháp đánh giá}

Ước lượng mưa sẽ được đánh giá bởi các chỉ số đánh giá bao gồm:

Sai số trung bình ME:

$$
M E=\frac{1}{n} \sum_{i=1}^{n}\left(F_{i}-O_{i}\right)
$$

Trong đó $F_{i}$ là giá trị dự báo, $O_{i}$ là giá trị quan trắc. $n$ là dung lượng mẫu.

Sai số quân phương RMSE:

$$
R M S E=\sqrt{\frac{1}{n} \sum_{i=1}^{n}\left(F_{\mathrm{i}}-O_{\mathrm{i}}\right)^{2}}
$$

RMSE chỉ bằng 0 khi giá trị dự báo bằng giá trị quan trắc tại mọi điểm trong không gian đánh giá.

\section{Kết quả và thảo luận}

3.1. Đánh giá uớc lượng mura cho hình thế gió mùa tây nam

Sau khi đối chiếu số liệu quan trắc radar với quan trắc mưa thực tế và loại bỏ các trường hợp mưa không có số liệu radar, kết quả thu được 21 trường hợp mưa thuộc hình thế gió mùa Tây nam của 14 trạm có đầy đủ cả số liệu quan trắc radar và quan trắc mưa thực tế. Giá trị lượng mưa trung bình của các đợt mưa thuộc hình thế này là $18.1 \mathrm{~mm}$.

Ta có thể thấy chỉ số $\mathrm{ME}$ của các công thức ước lượng mưa đã sử dụng, các phương pháp ước lượng khác có giá trị âm trong khi công thức ước lượng mưa được Nguyễn Hướng Điền đưa ra có giá trị dương.

Trong các công thức sử dụng, công thức ước lượng mưa diện rộng của Joss có giá trị RMSE nhỏ nhất tuy nhiên 2 giá trị này ít khác biệt với công thức của Marshall-Palmer. Từ đây ta có thể thấy với hình thế này, công thức mưa diện rộng của Joss là công thức tối ưu nhất cho hình thế mưa do gió mùa Tây nam với sai số trung bình $5 \mathrm{~mm} / \mathrm{h}$. 
Bảng 2. Chỉ số đánh giá của hình thế gió mùa Tây nam $(\mathrm{mm} / \mathrm{h})$

\begin{tabular}{lcc}
\hline & ME & RMSE \\
\hline VIL_5 & -5.44 & 12.63 \\
VIL_15 & -5.43 & 12.62 \\
MARSHALL & -5.25 & 12.11 \\
VNU & 2.29 & 17.5 \\
JOSS & -5.46 & 12.44 \\
JONES & -5.45 & 12.42 \\
Diện rộng & -5.24 & 12.09 \\
\hline
\end{tabular}

3.2. Đánh giá ước lượng mua cho hình thế dải hội tụ nhiệt đới

Sau khi đối chiếu và chọn lọc số liệu, thu được 33 trường hơp mưa do hình thế dải hội tụ nhiệt đới thuộc về 14 trạm trong khu vực Thành phố Hồ Chí Minh. Lượng mưa trung bình của các đợt mưa là $27.13 \mathrm{~mm}$.

Chỉ số ME của các công thức trong hình thế này đều mang dấu âm, cho thấy các giá trị ước lượng đều nhỏ hơn giá trị quan trắc thực tế. Công thức của Nguyễn Hướng Điền có chỉ số ME thiên âm khá nhỏ cho thấy công thức này thường cho giá trị ước lượng nhỏ hơn giá trị quan trắc.

Từ bảng 3 , trong tất cả các công thức ước lượng, công thức do Nguyễn Hướng Điền có sai số nhỏ nhất và là công thức tối ưu nhất để ước lượng mưa cho hình thế nàymặc dù giá trị sai số trung bình lên đến $9 \mathrm{~mm} / \mathrm{h}$. Các công thức khác có các chỉ số đánh giá có giá trị gần giống nhau và đưa ra ước lượng nhỏ hơn giá trị thực tế khoảng $15 \mathrm{~mm} / \mathrm{h}$.

\section{3. Đánh giá ước lương mưa cho hình thế $I T C Z+S W$}

Số liệu được sử dụng gồm 46 trường hợp mưa của 14 trạm được xác định là mưa do hình thế dải hội tụ nhiệt đới kết hợp với gió mùa tây nam. Lượng mưa trung bình các đợt mưa là $36.13 \mathrm{~mm}$.
Bảng 3. Các chỉ số đánh giá của hình thế dải hội tụ nhiệt đới $(\mathrm{mm} / \mathrm{h})$

\begin{tabular}{lcc}
\hline & ME & RMSE \\
\hline VIL_5 & -15.03 & 41.31 \\
VIL_15 & -15.03 & 41.3 \\
MARSHALL & -14.82 & 39.73 \\
VNU & -1.25 & 22.21 \\
JOSS & -14.96 & 40.67 \\
JONES & -14.98 & 40.71 \\
Diện rộng & -14.85 & 39.82 \\
\hline
\end{tabular}

Bảng 4. Các chỉ số đánh giá của hình thế ITCZ+SW (mm/h)

\begin{tabular}{lcc}
\hline & ME & RMSE \\
\hline VIL_5 & -28.11 & 41.31 \\
VIL_15 & -28.1 & 41.3 \\
MARSHALL & -27.08 & 39.73 \\
VNU & 7.29 & 22.21 \\
JOSS & -27.77 & 40.67 \\
JONES & -27.81 & 40.71 \\
Diện rộng & -27.14 & 39.82 \\
\hline
\end{tabular}

Chỉ số ME của công thức ước lượng do Nguyễn Hướng Điền đưa ra có giá trị dương trong khi các công thức khác có giá trị âm lớn. Điều này chứng tỏ các công thức trên thường cho giá trị ước lượng nhỏ hơn giá trị quan trắc trong khi công thức của Nguyễn Hướng Điền thường cho giá trị cao hơn giá trị quan trắc.

Dựa vào phân tích chỉ số ME, RMSE, ta có thể thấy trong 5 công thức ước lượng, công thức của Nguyễn Hướng Điền là tối ưu nhất với RMSE chỉ bằng một nửa các công thức khác. Công thức do Nguyễn Hướng Điền đưa ra đã có cải tiến so với các công thức cũ, tuy vậy, sai số của công thức này vẫn còn khá cao, lên đến $14 \mathrm{~mm} / \mathrm{h}$.

3.4. Đánh giá ước lượng mưa cho hình thế vùng áp thấp

Số liệu sử dụng để đánh giá ước lượng mưa cho hình thế này gồm 18 trường hợp mưa của 
14 trạm trong khu vực. Lượng mưa trung bình các đợt mưa là $20.94 \mathrm{~mm}$.

Chỉ số ME của các công thức đều có giá trị âm, các công thức này đều cho giá trị ước lượng mưa nhỏ hơn giá trị mưa thực tê.

Các chỉ số ME, RMSE của các công thức Marshall-Palmer, 2 công thức ước lượng mưa của Joss, công thức ước lượng mưa của Jones và ước lượng mưa qua VIL đều có giá trị giống nhau.

Trong khi đó công thức do Nguyễn Hướng Điền đưa ra có giá trị các chỉ số đánh giá thấp hơn nhiều so với các công thức khác, từ đó có thể kêt luận công thức ước lượng mưa này là công thức phù hợp nhất để ước lượng mưa cho hình thế vùng áp thấp với sai số khoảng $9.6 \mathrm{~mm} / \mathrm{h}$.

Bảng 5. Các chỉ số đánh giá của hình thế vùng áp thấp $(\mathrm{mm} / \mathrm{h})$

\begin{tabular}{lcc}
\hline & ME & RMSE \\
\hline VIL_5 & -13.65 & 15.53 \\
VIL_15 & -13.65 & 15.53 \\
MARSHALL & -13.63 & 15.51 \\
VNU & -5.25 & 9.75 \\
JOSS & -13.64 & 15.52 \\
JONES & -13.65 & 15.53 \\
Diện rộng & -13.64 & 15.52 \\
\hline
\end{tabular}

Bảng 6. Các chỉ số đánh giá của hình thế dông nhiệt $(\mathrm{mm} / \mathrm{h})$

\begin{tabular}{lcc}
\hline & ME & RMSE \\
\hline VIL_5 & -34.31 & 51.84 \\
VIL_15 & -34.19 & 51.69 \\
MARSHALL & -34.47 & 51.86 \\
VNU & -12.7 & 30.96 \\
JOSS & -34.84 & 52.46 \\
JONES & -34.87 & 52.51 \\
Diện rộng & -34.52 & 51.93 \\
\hline
\end{tabular}

\section{5. Đánh giá wớc luợng mưa cho hình thế dông nhiệt}

Hình thế này được đánh giá qua 19 trường hợp mưa được xác định tại 14 trạm trong khu vực Thành phố Hồ Chí Minh. Giá trị lượng mưa trung bình là $57.4 \mathrm{~mm}$. Chỉ số $\mathrm{ME}$ của các công thức đều mang giá trị âm. Các giá trị này đều rất lớn, nhỏ nhất là của công thức do Nguyễn Hướng Điền đưa ra cũng có giá trị $\mathrm{ME}$ và RMSE lần lượt là -12.7 và $30.96 \mathrm{~mm} / \mathrm{h}$.

Ta có thể thấy được công thức Nguyễn Hướng Điền đưa ra có ước lượng mưa tốt nhất trong các công thức. Tuy nhiên với sai số trung bình lên đến $18.9 \mathrm{~mm} / \mathrm{h}$ chỉ số RMSE là 30.96 $\mathrm{mm} / \mathrm{h}$ thì cần có cải tiến để có thể đưa ra ước lượng mưa chính xác hơn cho hình thế này.

3.6. Đánh giá ước luọng mua cho hình thế nhiễu động gió đông

Số liệu sử dụng để đánh giá gồm 26 trường hợp mưa được xác định do hình thế nhiễu động gió đông gây ra. Lượng mưa trung bình là $29.43 \mathrm{~mm}$.

Chỉ số $\mathrm{ME}$ của các công thức đều có giá trị âm, riêng công thức do Nguyễn Hướng Điền đưa ra có giá trị dương. Các công thức kia đều cho giá trị ước lượng mưa nhỏ hơn giá trị mưa thực tế trong khi công thức Nguyễn Hướng Điền đưa ra hay cho giá trị ước lượng cao hơn thực tế.

Ta có thể thấy qua biểu đồ các chỉ số $\mathrm{ME}$ và RMSE của công thức ước lượng mưa do Nguyễn Hướng Điền đưa ra có giá trị thấp hơn các công thức khác nên có thể dùng để ước lượng mưa cho hình thế nhiễu động gió đông với sai số trung bình khoảng $8 \mathrm{~mm} / \mathrm{h}$ (bảng 7 ).

3.7. Đánh giá ước luợng mura cho hình thế xoáy thuận nhiệt đới kết hợp gió mùa tây nam $(X T N D+S W)$

Ước lượng mưa cho hình thế này được đánh giá qua 12 trường hợp mưa thu được từ 14 trạm trong khu vực thành phố Hồ Chí Minh với lượng mưa trung bình là $25.3 \mathrm{~mm}$ 
Chỉ số $\mathrm{ME}$ của các công thức đều âm cho thấy tất cả các công thức đều cho ước lượng mưa nhỏ hơn thực tế.Giá trị các chỉ số ME, RMSE của các công thức đều gần giống nhau trừ công thức đưa ra bởi Nguyễn Hướng Điền.

Ta có thể thấy chỉ số ME và RMSE của công thức đưa ra bởi Nguyễn Hướng Điền có giá trị chỉ bằng một nửa so với các công thức khác. Vậy nên ta có thể sử dụng công thức này để ước lượng mưa cho hình thế XTND+SW với sai số khoảng $8.5 \mathrm{~mm} / \mathrm{h}$ (bảng 8 ).

Bảng 7. Các chỉ số đánh giá của hình thế nhiễu động gió đông $(\mathrm{mm} / \mathrm{h})$

\begin{tabular}{lcc}
\hline & ME & RMSE \\
\hline VIL_5 & -10.91 & 18.32 \\
VIL_15 & -10.89 & 18.31 \\
MARSHALL & -10.64 & 18.03 \\
VNU & 0.44 & 10.75 \\
JOSS & -10.9 & 18.38 \\
JONES & -10.89 & 18.38 \\
Diện rộng & -10.69 & 18.13 \\
\hline
\end{tabular}

Bảng 8. Các chỉ số đánh giá của hình thế $\mathrm{XTND}+\mathrm{SW}(\mathrm{mm} / \mathrm{h})$

\begin{tabular}{lcc}
\hline & ME & RMSE \\
\hline VIL_5 & -14.97 & 18.9 \\
VIL_15 & -14.96 & 18.9 \\
MARSHALL & -14.96 & 18.89 \\
VNU & -6.18 & 12.17 \\
JOSS & -14.98 & 18.91 \\
JONES & -14.98 & 18.91 \\
Diện rộng & -14.96 & 18.9 \\
\hline
\end{tabular}

3.8. Đánh giá uớc lượng mưa cho hình thế rãnh thấp có trục tây bắc - đông nam (TB - ĐN)

Đây là một hình thế hiếm khi xuất hiện nên trong khuôn khổ số liệu sử dụng cho bài báo chỉ chọn lọc được 5 trường hợp mưa lớn do hình thế này gây nên. Lượng mưa trung bình của các đợt mưa này là $19.4 \mathrm{~mm}$.
Bảng 9. Các chỉ số đánh giá của hình thế TB-ĐN (mm/h)

\begin{tabular}{lcc}
\hline & ME & RMSE \\
\hline VIL_5 & -13.55 & 16.53 \\
VIL_15 & -13.55 & 16.53 \\
MARSHALL & -13.54 & 16.51 \\
VNU & -8.1 & 11.87 \\
JOSS & -13.55 & 16.52 \\
JONES & -13.55 & 16.53 \\
Diện rộng & -13.54 & 16.52 \\
\hline
\end{tabular}

Chỉ số $\mathrm{ME}$ của các công thức mang giá trị âm chứng tỏ các công thức đều cho giá trị ước lượng mưa nhỏ hơn lượng mưa thực tế.

Qua biểu đồ ta có thể thấy công thức ước lượng mưa được đưa ra bởi Nguyễn Hướng Điền là ước lượng mưa tối ưu cho hình thế này.Với giá trị RMSE chỉ $11.87 \mathrm{~mm} / \mathrm{h}$ và sai số trung bình vào khoảng $9.5 \mathrm{~mm} / \mathrm{h}$.

\section{9. Đánh giá ước lương mưa cho các hình thế khác}

Dưới đây là đánh giá ước lượng mưa của các trường hợp mưa lớn không thể xếp loại vào các hình thế đã kể ở trên. Ước lượng mưa được đánh giá từ 41 trường hợp mưa lớn từ số liệu 14 trạm trong khu vực thành phố Hồ Chí Minh. Lượng mưa trung bình của các đợt mưa là $43.69 \mathrm{~mm}$.

Chỉ số ME của các công thức đều mang giá trị âm chứng tỏ các ước lượng mưa đều cho giá trị nhỏ hơn giá trị mưa thực tế.Tuy nhiên ta có thể thấy chỉ số $\mathrm{ME}$ của công thức Nguyễn Hướng Điền đưa ra có giá trị $\mathrm{ME}$ rất nhỏ $(-0.5)$ cho thấy công thức này có phần nhiều cho giá trị ước lượng nhỏ hơn thực tế tuy nhiên cũng không ít lần cho giá trị cao hơn.

Với chỉ số RMSE có giá trị chỉ bằng một nửa các công thức ước lượng mưa khác, công thức được Nguyễn Hướng Điền đưa ra là công thức tối ưu để ước lượng mưa với các hình thế khác không phổ biến này mặc dù sai số lên đến $14.7 \mathrm{~mm} / \mathrm{h}$. 
Bảng 10. Các chỉ số đánh giá của các hình thế khác $(\mathrm{mm} / \mathrm{h})$

\begin{tabular}{lcc}
\hline & ME & RMSE \\
\hline VIL_5 & -28.2 & 37.6 \\
VIL_15 & -28.2 & 37.6 \\
MARSHALL & -27.6 & 37 \\
VNU & -0.5 & 20.8 \\
JOSS & -28.1 & 37.4 \\
JONES & -28.1 & 37.5 \\
Diện rộng & -27.7 & 37 \\
\hline
\end{tabular}

\section{Kết luận}

So sánh kết quả nghiên cứu của Nguyễn Hướng Điền (2015) [2], kết quả nghiên cứu bài báo này cho kết quả tương đồng, các chỉ số đánh giá của các hình thế trong bài này có giá trị cao hơn với chỉ số $\mathrm{ME}$ gần giống nhất thuộc về hình thế mưa do nhiễu động gió đông, trong khi chỉ số RMSE gần giống nhất lại thuộc về hình thế mưa do vùng áp thấp.

Trong số 6 công thức ước lượng được sử dụng, công thức của ước lượng mưa diện rộng của Joss và công thức của Marshall-Plamer có thể được sử dụng để ước lượng mưa cho hình thế gió mùa tây nam đơn thuần với sai số vào khoảng là $5 \mathrm{~mm} / \mathrm{h}$ và thường nhỏ hơn giá trị quan trắc.

Công thức ước lượng mưa do Nguyễn Hướng Điền đưa ra là ước lượng tối ưu cho các hình thế gây mưa còn lại gồm: hình thế dải hội tụ nhiệt đới, hình thế gió mùa tây nam kết hợp dải hội tụ nhiệt đới, hình thế vùng áp thấp đơn thuần, hình thế dông nhiệt, hình thế nhiễu động gió đông, vùng áp thấp kết hợp với gió mùa tây nam, hình thế rãnh áp thấp có hướng tây bắc- đông nam và các hình thế khác với sai số lần lượt là $9.5 \mathrm{~mm} / \mathrm{h}, 14 \mathrm{~mm} / \mathrm{h}, 7 \mathrm{~mm} / \mathrm{h}, 19 \mathrm{~mm} / \mathrm{h}$, $8 \mathrm{~mm} / \mathrm{h}, 8.5 \mathrm{~mm} / \mathrm{h}, 9.5 \mathrm{~mm} / \mathrm{h}$ và $14.7 \mathrm{~mm} / \mathrm{h}$.

Công thức ước lượng mưa theo VIL vẫn chưa được chính xác do trong công thức không tính đến việc cột nước trong khí quyển được bổ sung lượng ẩm trong giai đoạn hình thành, phát triển và chín muồi của đám mây đối lưu. Việc này dẫn đến một số thời điểm có lượng ước lượng mưa bằng 0 trong khi thực tế có mưa.

\section{Lời cảm ơn}

Nghiên cứu này được thực hiện và hoàn thành nhờ sự hỗ trợ của Đề tài "Xây dựng hệ thống nghiệp vụ dự báo mưa lớn hạn cực ngắn trên địa bàn Thành phố Hồ Chí Minh".Tác giả xin chân thành cảm ơn.

\section{Tài liệu tham khảo}

[1] Nguyễn Hướng Điền, 2015. Công thức thực nghiệm tính toán cường độ mưa từ độ phản hồi radar cho khu vực Đông Nam Bộ. Tạp chí Khoa học ĐHQGHN: Khoa học Tự nhiên và Công nghệ, Tập 31, Số 3S tr.66 -70.

[2] WMO/ASEAN Training Workshop on Weather Radar Data Quality and Standardization held in Bangkok, Thailand, from 5 to 13 February 2018

[3] Battan, L.J., 1973. Radar observation of the atmosphere. The University of Chicago Press, Chicago, 324 pp.

[4] J. S. Marshall and W. McK. Palmer (1946), "The Distribution of Raindrop with Size", Journal of Meteorology, 5, pp. 165-166.

[5] J. Joss, Karin Schram, J. C. Thams and A. Waldvogel (1970), "On the Quantitative Determination of Precipitetion by a Radar", Issue 63 of Wissenschaftliche Mitteilung.

[6] D. Jones (1956), "Rainfall Drop Size-distribution and Radar Reflectivity”, Research Report No. 6. 


\title{
Assessing the Rain Estimate from the Feflectivity of Nha Be Radar
}

\author{
Cong Thanh ${ }^{1}$, Nguyen Nhu Quy ${ }^{1}$, Mai Van Khiem ${ }^{2}$ \\ ${ }^{I}$ Faculty of Hydro-Meteorology and Oceanography, VNU Hanoi University of Science, \\ 334 Nguyen Trai, Thanh Xuan, Hanoi, Vietnam \\ ${ }^{2}$ Viet Nam Institute of Meteorology Hydrology and Climate Change, \\ 23/62 Nguyen Chi Thanh, Dong Da, Hanoi, Vietnam
}

\begin{abstract}
This study evaluates the estimated rainfall from Nha Be radar observation using different experimental formulas. The results show that in six estimation formulas used, the formulation of Joss' large-scale rainfall estimation and Marshall-Plamer's formulation can be used to estimate precipitation for the southwest monsoon with an error of approximately $5 \mathrm{~mm} / \mathrm{h}$ and usually smaller than the observed value. The rainfall estimation formula by Nguyen Huong Dien is the optimum estimation for the 9 synoptic rainfall patterns: Intertropical Convergence Zone, Southwest monsoon associated with Intertropical Convergence Zone, low-pressure area, thunderstorm, disturbance in East winds, low-pressure area associated with Southwest monsoon, low-pressure troughs have a Northwestern-Southeasterly direction and other synoptic patterns with errors: $5 \mathrm{~mm} / \mathrm{h}, 9.5 \mathrm{~mm} / \mathrm{h}, 14$ $\mathrm{mm} / \mathrm{h}, 7 \mathrm{~mm} / \mathrm{h}, 19 \mathrm{~mm} / \mathrm{h}, 8 \mathrm{~mm} / \mathrm{h}, 8.5 \mathrm{~mm} / \mathrm{h}, 9.5 \mathrm{~mm} / \mathrm{h}$ and $14.7 \mathrm{~mm} / \mathrm{h}$, respectively.
\end{abstract}

Keywords: Radar, rain estimate. 